\title{
Fenómenos de envejecimiento y oxidación a altas temperaturas en tres aceros inoxidables altamente aleados $^{(\bullet)}$
}

\author{
J. Botella $^{(*)}$, J. Almagro ${ }^{(*)}$, E. Otero ${ }^{(* *)}$, P. Hierro ${ }^{(* *)}$ y C. Merino ${ }^{(* *)}$ \\ Resumen Se parte de tres aceros inoxidables austeníticos refractarios $\left(25 \mathrm{Cr} 20 \mathrm{Ni}, 21 \mathrm{Cr} 11 \mathrm{Ni}_{(1)}, 21 \mathrm{Cr} 11 \mathrm{Ni}_{(2)}\right)$ y \\ se estudian las cinéticas de oxidación al aire, hasta 50 h a 1.373 y $1.473 \mathrm{~K}$. El mejor comportamiento \\ a $1.373 \mathrm{~K}$ corresponde al acero $25 \mathrm{Cr} 20 \mathrm{Ni}$ seguido de cerca del $21 \mathrm{Cr} 11 \mathrm{Ni}_{(1)}$ con $0,036 \%$ de lantáni- \\ dos, mientras que el peor comportamiento corresponde al $21 \mathrm{Cr} 11 \mathrm{Ni}_{(2)}$ con $0,010 \%$ de lantánidos. A \\ $1.473 \mathrm{~K}$ también se comporta mejor el $25 \mathrm{Cr} 20 \mathrm{Ni}$, a distancia de los otros inoxidables. Un tratamiento \\ de oxidación al aire de $2 \mathrm{~h}$ a $1.323 \mathrm{~K}$ da lugar a capas de oxidación de 1 a $3 \mu \mathrm{m}$ de espesor en los tres \\ materiales y, en el examen de las capas de óxidos mediante microscopía electrónica de barrido \\ (MEB), microanálisis por dispersión de energías de rayos X (EDX) y difracción de rayos X (DRX), \\ se observan las mismas características en los tres aceros: espinelas tipo $\mathrm{MnCr}_{2} \mathrm{O}_{4}$ en el exterior, \\ $\mathrm{Cr}_{2} \mathrm{O}_{3}$ en el interior, con barreras de $\mathrm{SiO}_{2}$ én la interfase óxido-metal y precipitados internos e inter- \\ granulares (también de $\mathrm{SiO}_{2}$ ) muy abundantes en los aceros $21 \mathrm{Cr} 11 \mathrm{Ni}$. Los ensayos de envejecimien- \\ to de $400 \mathrm{~h}$ a $1.073 \mathrm{~K}$ producen en el acero $25 \mathrm{Cr} 20 \mathrm{Ni}$ (AISI $310 \mathrm{~S}$ ) alrededor de un $10 \%$ vol. de fase \\ $\sigma$, mientras que los aceros $21 \mathrm{Cr} 11 \mathrm{Ni}$ o no la forman o lo hacen en muy pequeña cantidad, pudiendo \\ influir en ello los elevados niveles de nitrógeno de estos últimos. La aplicación de la MEB con fila- \\ mento de emisión de campo (EC) con EDX permite el análisis de la fase $\sigma$ así como de los carburos, \\ nitruros y carbonitruros presentes. $\mathrm{El}$ acero $25 \mathrm{Cr} 20 \mathrm{Ni}$ sólo forma, junto con $\sigma$, carburos tipo $\mathrm{M}_{23} \mathrm{C}_{6}$, \\ mientras que el acero $21 \mathrm{Cr} 11 \mathrm{Ni}_{(1)}$ forma estos carburos y nitruros $\mathrm{M}_{15} \mathrm{~N}\left(\mathrm{M}_{8} \mathrm{~N}\right.$ en la superficie) y el \\ acero $21 \mathrm{Cr} 11 \mathrm{Ni}_{(2)}$ preferentemente forma $\mathrm{M}_{6}(\mathrm{CN})$. Esta diferencia de comportamiento de los aceros \\ $21 \mathrm{Cr} 11 \mathrm{Ni}$ también puede ser imputable a los niveles de nitrógeno relativamente mayores de la alea- \\ ción $21 \mathrm{Cr} 11 \mathrm{Ni}_{(1)}$.
}

Palabras clave: Aceros inoxidables. Austeníticos refractarios. Oxidación al aire. Altas temperaturas. Envejecimiento térmico. Fase $\sigma$. Precipitados.

\section{Ageing and high temperature oxidation phenomena in three high alloyed stainless steels}

\begin{abstract}
The oxidation kinetics in air up to $50 \mathrm{~h}$ at 1,373 and $1,473 \mathrm{~K}$ of three refractory stainless steels $\left(25 \mathrm{Cr} 20 \mathrm{Ni}, 21 \mathrm{Cr} 11 \mathrm{Ni}_{(1)}, 21 \mathrm{Cr} 11 \mathrm{Ni}_{(2)}\right)$ are studied. At $1,373 \mathrm{~K}$, the best results are those of $25 \mathrm{Cr} 20 \mathrm{Ni}$ followed by those of $21 \mathrm{Cr}_{11} 1 \mathrm{Ni}_{(1)}$, with $0,036 \%$ lanthanides, and the worst behaviour is that of $21 \mathrm{Cr} 11 \mathrm{Ni}_{(2)}$, with $0,010 \%$ lanthanides. A $2 \mathrm{~h}$ oxidation treatment at $1,323 \mathrm{~K}$ in air produces oxidation layers from 1 to $3 \mu \mathrm{m}$ in thickness for the three materials. Scanning electron microscopy and energy dispersive microanalysis (SEM - EDX) and X-ray diffraction (XRD) characterisation of the oxidation layers give the same characteristics for the three steels: $\mathrm{MnCr}_{2} \mathrm{O}_{4}$ spinel type in the outer part, $\mathrm{Cr}_{2} \mathrm{O}_{3}$ in the inner one, with a $\mathrm{SiO}_{2}$ barrier in the oxide-metal interface and a large amount of internal and intergranular precipitates $\left(\right.$ also $\left.\mathrm{SiO}_{2}\right)$ in $21 \mathrm{Cr} 11 \mathrm{Ni}$ materials. After ageing tests, at $1,073 \mathrm{~K}$ for $400 \mathrm{~h}$, about 10 vol. $\%$ of the $\sigma$-phase is present in the $25 \mathrm{Cr} 20 \mathrm{Ni}$ (AISI $310 \mathrm{~S}$ ) material, while no $\sigma$-phase is present in the $21 \mathrm{Cr} 11 \mathrm{Ni}$ materials perhaps due to the high nitrogen content. The application of field emission SEM with EDX allows the analysis of $\sigma$-phase and carbides, nitrides and carbonitrides present. The steel $25 \mathrm{Cr} 20 \mathrm{Ni}$ only forms, in addition to the $\sigma$-phase, $\mathrm{M}_{23} \mathrm{C}_{6}$ type
\end{abstract}

$(\bullet \quad$ Trabajo recibido el día 21 de noviembre de 1997 y aceptado en su forma final el 23 de octubre de 1998.

(*) Acerinox, S.A. Centro de Investigación y Ensayos. 11379 Palmones, Los Barrios (Cádiz, España).
(**) Dpto. de Ciencia de los Materiales e Ingeniería Metalúrgica. Facultad de Ciencias Químicas. Universidad Complutense. 28040-Madrid (España). 
carbides, while the steel $21 \mathrm{Cr} 11 \mathrm{Ni}_{(1)}$ forms these carbides and $\mathrm{M}_{15} \mathrm{~N}$ nitrides $\left(\mathrm{M}_{8} \mathrm{~N}\right.$ in the surface) and the steel $21 \mathrm{Cr} 11 \mathrm{Ni}_{(2)}$ forms mainly carbonitrides $\mathrm{M}_{6}(\mathrm{CN})$. The relatively higher nitrogen level in the $21 \mathrm{Cr} 11 \mathrm{Ni}_{(1)}$ alloy could explain the different behaviour of both $21 \mathrm{Cr} 11 \mathrm{Ni}$ steels.

Keywords: Stainless steels. Refractory austenitic steels. Air oxidation. High temperature. Thermal ageing. $\sigma$-phase. Precipitates.

\section{INTRODUCCIÓN}

El diseño de aceros inoxidables para aplicaciones que implican resistencia a la oxidación al aire a altas temperaturas, pasa necesariamente por un conocimiento lo más profundo posible de cómo se constituyen las capas de óxidos, de sus composiciones químicas, sus estructuras cristalinas, las cinéticas de oxidación, los mecanismos de difusión puestos en juego, etc. Todos estos factores, y muchos más que tienen que ver con las propiedades mecánicas y estructurales a altas temperaturas, permiten la adecuación de un material a un uso refractario específico.

Pero conviene tener en cuenta que los materiales empleados a determinadas temperaturas pueden verse sometidos a condiciones diferentes, de forma ocasional o sistemática. Por ejemplo, en los ciclos de calentamiento, los materiales implicados alcanzan temperaturas por encima de las de régimen, durante tiempos variables, como consecuencia de las inercias térmicas. En los de enfriamiento, los aceros permanecen a temperaturas por debajo de las de uso nominal durante tiempos muy variables. En otras ocasiones, las ingenierías precisan de materiales aplicables en ambientes oxidantes a altas temperaturas (por ej. $1.273 \mathrm{~K}$ ) y que a la vez sean utilizables a temperaturas típicas de precipitación de carburos y de formación de fases fragilizantes (por ej. $1.073 \mathrm{~K}$ ).

En estas condiciones, es muy importante considerar que la aleación elegible está diseñada para superar las dificultades de la aplicación. El presente trabajo aporta resultados obtenidos experimentalmente en relación con los temas comentados anteriormente.

\section{PARTE EXPERIMENTAL}

Los materiales empleados han sido aceros inoxidables en forma de chapas comerciales.

La tabla I presenta la composición química y la tabla II muestra el espesor, el tamaño de grano y el estado metalúrgico de los tres aceros.

Se prepararon probetas de $50 \mathrm{~mm} \times 50 \mathrm{~mm}$ espesor, mediante corte con cizalla de las chapas originales y fresado refrigerado de los bordes hasta la total eliminación de las zonas afectadas por los cortes. Las superficies originales de las chapas se conservaron en las caras mayores de las probetas y éstas fueron dimensionadas usando un calibre y un micrómetro. Se calculó la superficie de cada probeta a partir de sus dimensiones y se pesaron con una balanza de precisión $0,0001 \mathrm{~g}$.

Se realizaron los tratamientos térmicos de oxidación mostrados en la tabla III.

TABLA I.- Composición química (\% en masa) de los aceros inoxidables

TABLE I.- Chemical composition (mass \%) of the stainless steels

\begin{tabular}{|l|c|c|c|c|c|c|c|}
\hline \multicolumn{1}{|c|}{ Materiales } & $\mathrm{Cr}$ & $\mathrm{Ni}$ & $\mathrm{Si}$ & $\mathrm{C}$ & $\mathrm{Mn}$ & $\mathrm{Cu}$ & $\mathrm{Mo}$ \\
\hline $25 \mathrm{Cr} 20 \mathrm{Ni}_{(\mathrm{AISI} 310 \mathrm{~S})}$ & 24,59 & 19,30 & 1,20 & 0,064 & 0,39 & 0,16 & 0,19 \\
$21 \mathrm{Cr} 11 \mathrm{Ni}_{(1)}$ & 20,91 & 10,90 & 1,47 & 0,087 & 0,48 & 0,27 & 0,18 \\
$21 \mathrm{Cr} 11 \mathrm{Ni}_{(2)}$ & 20,97 & 10,96 & 1,88 & 0,075 & 0,42 & 0,11 & 0,10 \\
\hline \multicolumn{1}{c|}{ Materiales $_{25 \mathrm{Cr} 20 \mathrm{Ni}_{(\mathrm{AISI} 310 \mathrm{~S})}}$} & $\mathrm{P}$ & $\mathrm{S}$ & $\mathrm{Ti}$ & $\mathrm{Co}$ & $\mathrm{N}$ & $\mathrm{V}$ & $\mathrm{Ln}$ 's \\
$21 \mathrm{Cr} 11 \mathrm{Ni}_{(1)}$ & 0,020 & 0,001 & 0,029 & 0,15 & 0,0247 & 0,089 & - \\
$21 \mathrm{Cr} 11 \mathrm{Ni}_{(2)}$ & 0,024 & 0,001 & 0,031 & 0,23 & 0,1367 & 0,115 & 0,010 \\
\hline
\end{tabular}

Notas: i.- $\quad$ El acero $25 \mathrm{Cr} 20 \mathrm{Ni}$ contiene además 0,0033,\% B

ii. - Ln's = representa la suma total de los porcentajes en masa correspondientes a tierras raras (La, Ce, etc.) 
TABLA II.- Espesores y características metalúrgicas de los aceros inoxidables

TABLE II.- Thickness and metallurgical characteristics of the stainless steels

\begin{tabular}{|l|c|c|l|}
\hline \multicolumn{1}{|c|}{ Materiales } & $\begin{array}{c}\text { Espesor } \\
(\mathrm{mm})\end{array}$ & $\begin{array}{c}\text { Tamaño de } \\
\text { grano } \\
(\mu \mathrm{m})\end{array}$ & \multicolumn{1}{|c|}{ Estado metalúrgico } \\
\hline $25 \mathrm{Cr} 20 \mathrm{Ni}$ (AISI 310 S) & 2,0 & 13 & $\begin{array}{l}\text { Laminado en caliente, recocido y } \\
\text { decapado, seguido de laminado en } \\
\text { frío, recocido y decapado. }\end{array}$ \\
\hline $21 \mathrm{Cr} 11 \mathrm{Ni}_{(1)}$ & 2,0 & 38 & $\begin{array}{l}\text { Laminado en caliente, recocido y } \\
\text { decapado, seguido de laminado en } \\
\text { frío, recocido y decapado. }\end{array}$ \\
\hline $21 \mathrm{Cr} 11 \mathrm{Ni}_{(2)}$ & 6,0 & 29 & $\begin{array}{l}\text { Laminado en caliente, recocido y } \\
\text { decapado. }\end{array}$ \\
\hline
\end{tabular}

TABLA III.- Tratamientos térmicos de oxidación

TABLE III.-Oxidation thermal treatments

\begin{tabular}{|c|c|c|c|c|c|c|c|c|c|c|c|c|}
\hline$T(\mathrm{~K})$ & \multicolumn{10}{|c|}{ Tiempo, $t(\mathrm{~h})$} \\
\hline 1.323 & - & - & - & 2 & - & - & - & - & - & - & - & - \\
1.373 & - & - & - & 2 & 3 & 5 & 8 & 10 & 20 & 30 & 40 & 50 \\
1.473 & 0,33 & 0,67 & 1 & 2 & 3 & 5 & 8 & 10 & 20 & 30 & 40 & 50 \\
\hline
\end{tabular}

Los tratamientos de oxidación se realizaron al aire en horno de mufla de calentamiento rápido, midiendo la temperatura de la zona próxima al soporte de las probetas con un termopar Pt/PtRh. A la temperatura de $1.323 \mathrm{~K}$ se realizaron seis ensayos de cada material y para 1.373 y $1.473 \mathrm{~K}$ se realizaron tres ensayos para cada pareja $T / t$.

Una vez transcurrido el tiempo a las distintas temperaturas, cada probeta se extrajo y se enfrió en un crisol de porcelana tapado para la recogida de los posibles óxidos desprendidos durante el enfriamiento y se determinó su ganancia de masa.

Los ensayos de envejecimiento, $400 \mathrm{~h}$ a 1.073 $\mathrm{K}$, se llevaron a cabo sobre probetas iguales a las de los ensayos de oxidación, y el tratamiento se llevó a cabo al aire en horno de mufla.

La constitución de las capas de óxidos se estableció mediante DRX (Siemens 5000) y MEB-EDX (Hitachi 570 - Kevex 8005, detector de Si(Li)). La DRX se hizo sobre las superficies mayores de las muestras y la MEB-EDX sobre las secciones metalográficas obtenidas, protegiendo los óxidos mediante vaporización de Pt (activación) y depositación electrolítica de níquel, antes del corte transversal y el proceso de pulido (1).

El estudio de la microestructura del material, después del tratamiento de envejecimiento, se reali- zó con un MEB-EC Zeiss DSM 982 GEMINI. Los análisis se realizaron con el sistema EDX incorporado (LINK ISIS 200 con detector de Ge).

\section{RESULTADOS Y DISCUSIÓN}

\subsection{Oxidación al aire a 1.373 y $1.473 \mathrm{~K}$}

Los cuadrados de los cocientes de las ganancias de masa por unidad de superficie frente a los tiempos de oxidación a cada temperatura se presentan en las figuras 1 y 2 . Las ecuaciones de correlación obtenidas entre $(\Delta \mathrm{m} / \mathrm{S})^{2}\left(\mathrm{mg}^{2} \mathrm{~cm}^{-4}\right)$ y $t(\mathrm{~h})$ se muestran en la tabla IV.

Parece evidente que, en las condiciones experimentales, los tres aceros presentan cinéticas asimilables a parabólicas para ambas temperaturas. A $1.373 \mathrm{~K}$, los comportamientos del $25 \mathrm{Cr} 20 \mathrm{Ni}$ y del $21 \mathrm{Cr} 11 \mathrm{Ni}_{(1)}$ son prácticamente iguales, oxidándose con mayor rapidez el $21 \mathrm{Cr} 11 \mathrm{Ni}_{(2)}$. A $1.473 \mathrm{~K} \mathrm{el}$ AISI $310 \mathrm{~S}$ tiene un excelente comportamiento, seguido del $21 \mathrm{Cr} 11 \mathrm{Ni}_{(1)}$. El mejor comportamiento del $21 \mathrm{Cr} 11 \mathrm{Ni}_{(1)}$ con respecto al $21 \mathrm{Cr} 11 \mathrm{Ni}_{(2)}$ posiblemente sea debido a los niveles superiores de lantánidos $\left(0,036 \%\right.$ para el $21 \mathrm{Cr} 11 \mathrm{Ni}_{(1)}$ frente a 0,010 $\%$ para el $\left.21 \mathrm{Cr} 11 \mathrm{Ni}_{(2)}\right)$. 


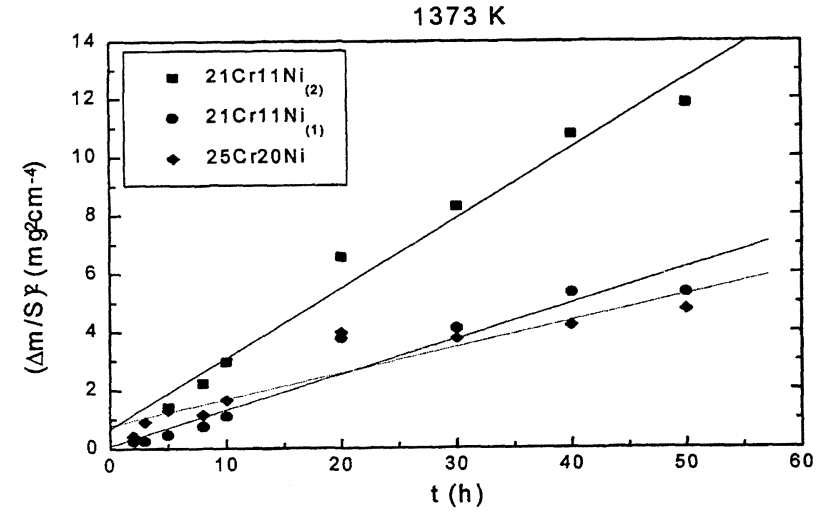

FIG. 1.- Variación del cuadrado del cociente de la ganancia de masa por unidad de superficie de los tres aceros durante el ensayo a $1.373 \mathrm{~K}$. Ajuste a una ecuación parabólica.

FIG. 1.- Variation of the square of the mass gain per surface unit rate, for the three steels during the 1,373 K test. Parabolic equation fitting.

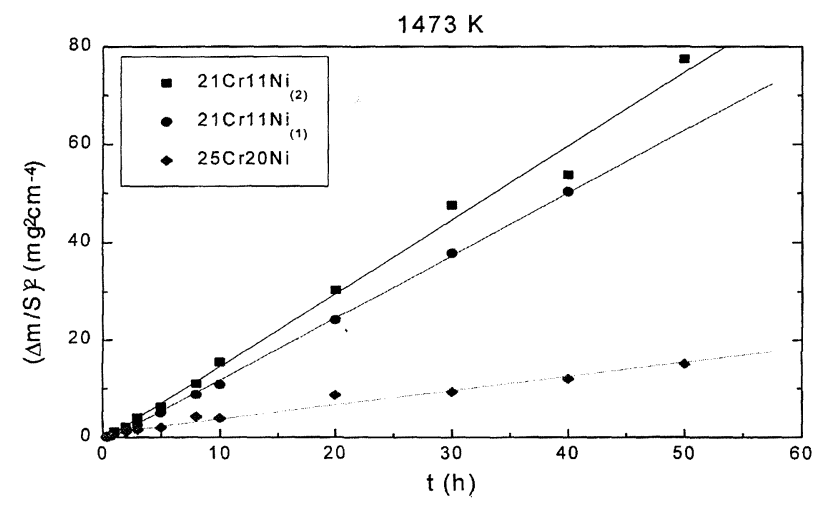

FIG. 2.- Variación del cuadrado del cociente de la ganancia de masa por unidad de superficie de los tres aceros durante el ensayo a $1.473 \mathrm{~K}$. Ajuste a una ecuación parabólica.

FIG. 2.- Variation of the square of the mass gain per surface unit rate, for the three steels during the 1,473 K test. Parabolic equation fitting.
TABla IV.- Ajustes parabólicos de las curvas termogravimétricas según la ecuación $(\Delta \mathrm{m} / \mathrm{s})^{2}=\mathrm{a} \mathrm{t} ;\left(\mathrm{mg}^{2} \mathrm{~cm}^{-4}\right)<>\mathrm{h}$

TABLE IV.- Parabolic fitting of thermogravimetric curves according to the equation

$(\Delta \mathrm{m} / \mathrm{s})^{2}=a t ;\left(\mathrm{mg}^{2} \mathrm{~cm}^{-4}\right)<>h$

\begin{tabular}{|l|c|c|}
\hline \multicolumn{1}{|c|}{ Materiales } & $\mathrm{a}(1.373 \mathrm{~K})$ & $\mathrm{a}(1.473 \mathrm{~K})$ \\
\hline $\left.25 \mathrm{Cr} 20 \mathrm{Ni}_{(\mathrm{AISI}} 310 \mathrm{~S}\right)$ & 0,3577 & 0,5839 \\
$21 \mathrm{Cr} 11 \mathrm{Ni}_{(1)}$ & 0,3547 & 1,1390 \\
$21 \mathrm{Cr} 11 \mathrm{Ni}_{(2)}$ & 0,5189 & 1,2104 \\
\hline
\end{tabular}

\subsection{Oxidación al aire a $1.323 \mathrm{~K}$}

Los resultados obtenidos se presentan en la tabla V.

De nuevo se pone de manifiesto el relativo mejor comportamiento del AISI $310 \mathrm{~S}$, aunque es muy parecido al del $21 \mathrm{Cr} 11 \mathrm{Ni}_{(1)}$.

En los tres materiales se forman capas de oxidación delgadas y continuas que reproducen las irregularidades de la rugosidad superficial del acero. La parte externa de las capas de óxidos presenta formas rectilíneas-angulares, típicas del corte de cristales de geometría bien definida. Las capas de óxidos tienen espesores entre 1 y $3 \mu \mathrm{m}$, cifras acordes con las calculadas a partir de los datos de $\Delta \mathrm{m} / \mathrm{S}$ (Tabla V).

Existe una diferencia importante en las morfologías de los óxidos del AISI $310 \mathrm{~S}$ y de los materiales $21 \mathrm{Cr} 11 \mathrm{Ni}$ : en éstos últimos son muy abundantes los óxidos internos y barreras intergranulares dentro del metal inmediatamente debajo de la capa de óxidos, tal como se muestra en las figuras 3 y 4 (imágenes de electrones retrodispersados, IER).

Por lo demás, los análisis en línea de las capas de óxidos de los tres aceros no muestran diferencias substanciales. Las figuras 5 y 6 , relativas al AISI $310 \mathrm{~S}$, son representativas y se dan como ejemplo: la línea atraviesa la capa de niquelado de izquierda a derecha, hasta llegar al Pt, frontera de un óxido de

TABLA V.- Resultados de oxidación después de 2 h a $1.323 \mathrm{~K}$

TABLE V.-Oxidation figures after $2 \mathrm{~h}$ at $1,323 \mathrm{~K}$

\begin{tabular}{|l|c|c|c|}
\hline \multirow{2}{*}{ Materiales } & \multicolumn{2}{|c|}{$\Delta \mathrm{m} / \mathrm{S}\left(\mathrm{mg} / \mathrm{cm}^{2}\right)$} & \multirow{2}{*}{$\begin{array}{c}\text { Espesor teórico de la } \\
\text { capa de óxidos }(\mu \mathrm{m})\end{array}$} \\
\cline { 2 - 3 } & $\overline{\mathrm{X}}$ & $\sigma_{\mathrm{n}-1}(\mathrm{n}=6)$ & 1,8 \\
\hline $25 \mathrm{Cr} 20 \mathrm{Ni}_{(\mathrm{AISI} 310 \mathrm{~S})}$ & 0,28 & 0,04 & 2,0 \\
$21 \mathrm{Cr} 11 \mathrm{Ni}_{(1)}$ & 0,32 & 0,04 & 3,0 \\
$21 \mathrm{Cr} 11 \mathrm{Ni}_{(2)}$ & 0,47 & 0,02 & \\
\hline
\end{tabular}




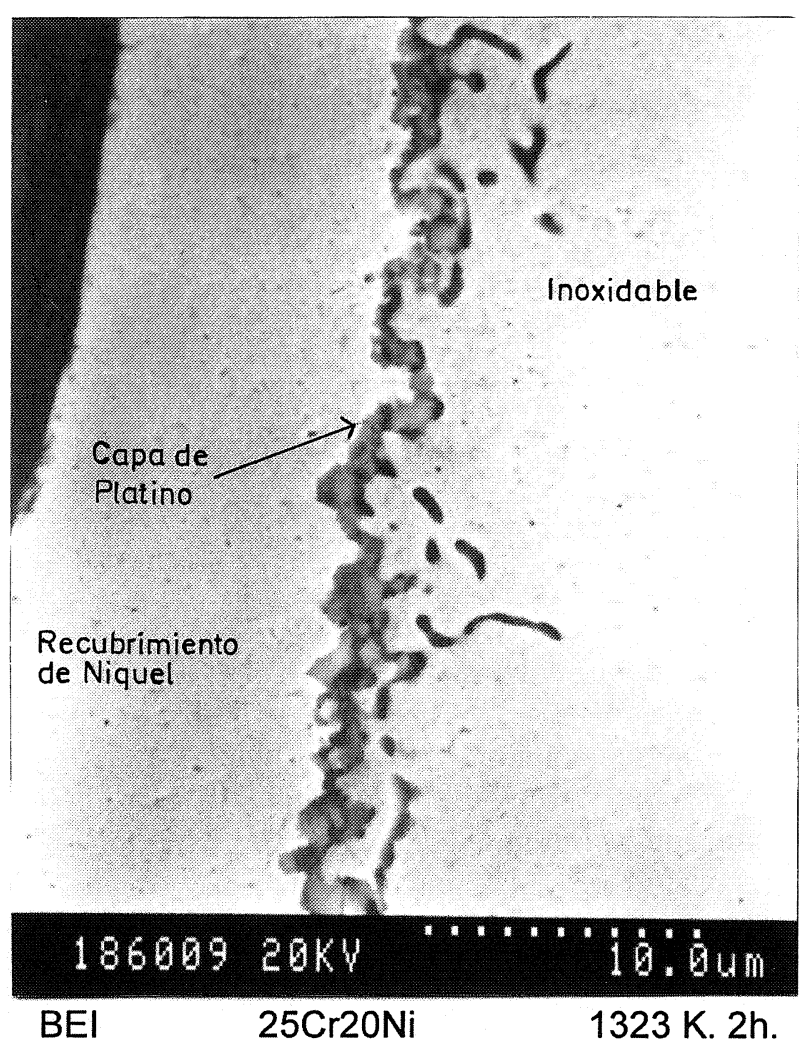

FIG. 3.- Corte transversal de los óxidos. Relativa baja rugosidad del acero.

FIG. 3.- Surface oxide cross section. Relative low roughness of the steel.

forma claramente hexagonal cuyo borde atmosférico tiene como componentes manganeso y cromo, deduciéndose de los microanálisis y de la DRX que los óxidos más externos pudieran ser espinelas tipo $\mathrm{MnCr}_{2} \mathrm{O}_{4}$ con una pequeña participación de cobre. Hacia el interior, prácticamente toda la oblea hexagonal es de $\mathrm{Cr}_{2} \mathrm{O}_{3}$, con algo de vanadio; la interfase óxido-metal aparece enriquecida con $\mathrm{SiO}_{2}$, así como también los precipitados internos son de $\mathrm{SiO}_{2}$. Las formaciones ricas en silicio están precedidas, en el sentido atmósfera-aleación, por una zona enriquecida con molibdeno.

\subsection{Envejecimiento 400 h a $1.073 \mathrm{~K}$}

Las micrografías de las figuras 7, 8 y 9 son imágenes de electrones retrodispersados y presentan aspectos de las fases y precipitados observables en cada uno de los materiales tras el envejecimiento. Un aspecto relevante es que en el $21 \mathrm{Cr} 11 \mathrm{Ni}_{(1)}$ no se observa fase $\sigma$, siendo escasa su presencia en el $21 \mathrm{Cr} 11 \mathrm{Ni}_{(2)}$ y relativamente abundante en el AISI $310 \mathrm{~S}$. En este caso resulta difícil la cuantificación debido a la heterogeneidad en la distribución en el espesor: próximo al borde, en la interfase óxidosmatriz metálica, existe una zona de 5 a $10 \mu \mathrm{m}$ de

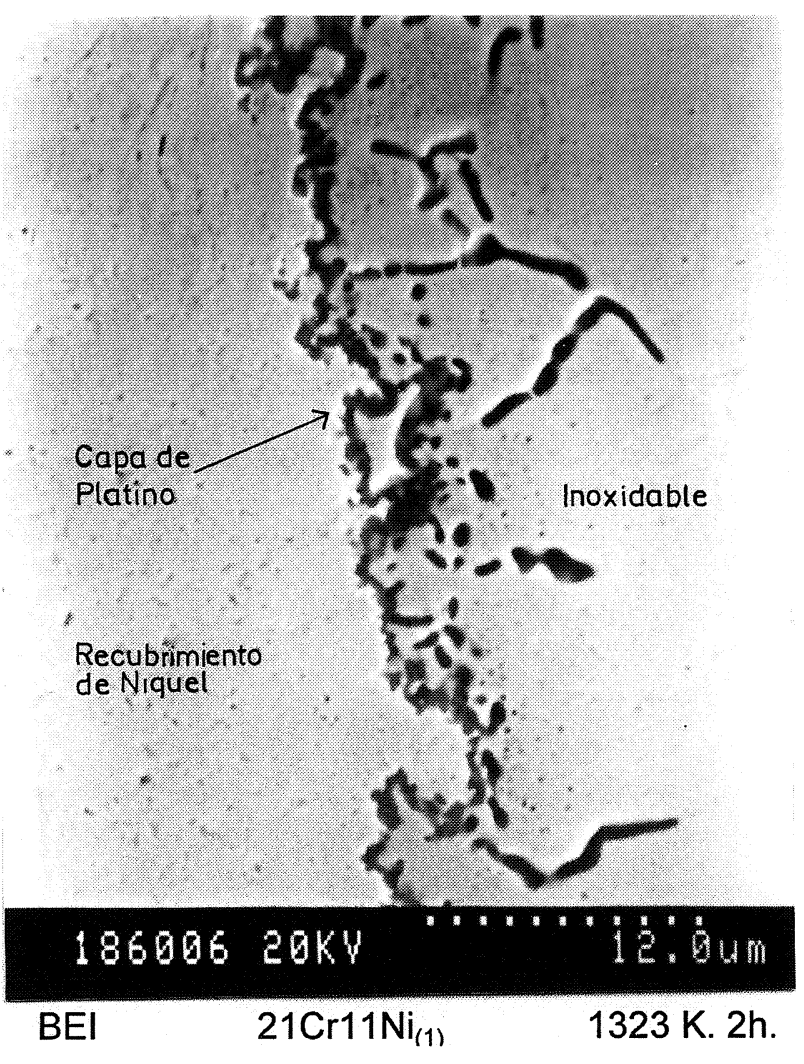

FIG. 4.- Corte transversal de los óxidos. Relativa alta rugosidad del inoxidable. Precipitados internos muy abundantes, muchos intergranulares.

Fig. 4.- Surface oxide cross section. Relative high roughness of the steel. A large amount of internal precipitates, mainly following the grain boundary.

espesor que está prácticamente libre de presencia de fase $\sigma$, posiblemente debido al empobrecimiento de cromo de esa región, por el gradiente de composición química originado en la formación de óxidos superficiales ricos en $\mathrm{Cr}_{2} \mathrm{O}_{3}$. La máxima presencia de fase $\sigma$ se sitúa en el centro del espesor en donde, inicialmente, el material presentaba residuos de la ferrita delta de solidificación que no habían sido disueltos totalmente por las transformaciones termomecánicas de la laminación en caliente.

A pesar de las dificultades comentadas de las heterogeneidades en el reparto de la fase $\sigma$ es posible decir que, tras $400 \mathrm{~h}$ a $1.073 \mathrm{~K}$, el AISI $310 \mathrm{~S}$ forma en torno al $10 \%$ vol. de fase $\sigma$, mientras que sólo hay una mínima presencia, inferior al $1 \%$, en el $21 \mathrm{Cr} 11 \mathrm{Ni}_{(2)}$. La tabla VI ofrece las composiciones químicas de las fases $\sigma$ encontradas en los dos aceros en cuestión, así como sus estequiometrías probables. La tabla VII ofrece las relaciones entre los porcentajes en masa de los elementos en las fases $\sigma$ y en las matrices originales, comparando dichos cocientes con los de otros trabajos (2).

La tabla VIII compendia los resultados analíticos de carburos, nitruros y carbonitruros presentes 


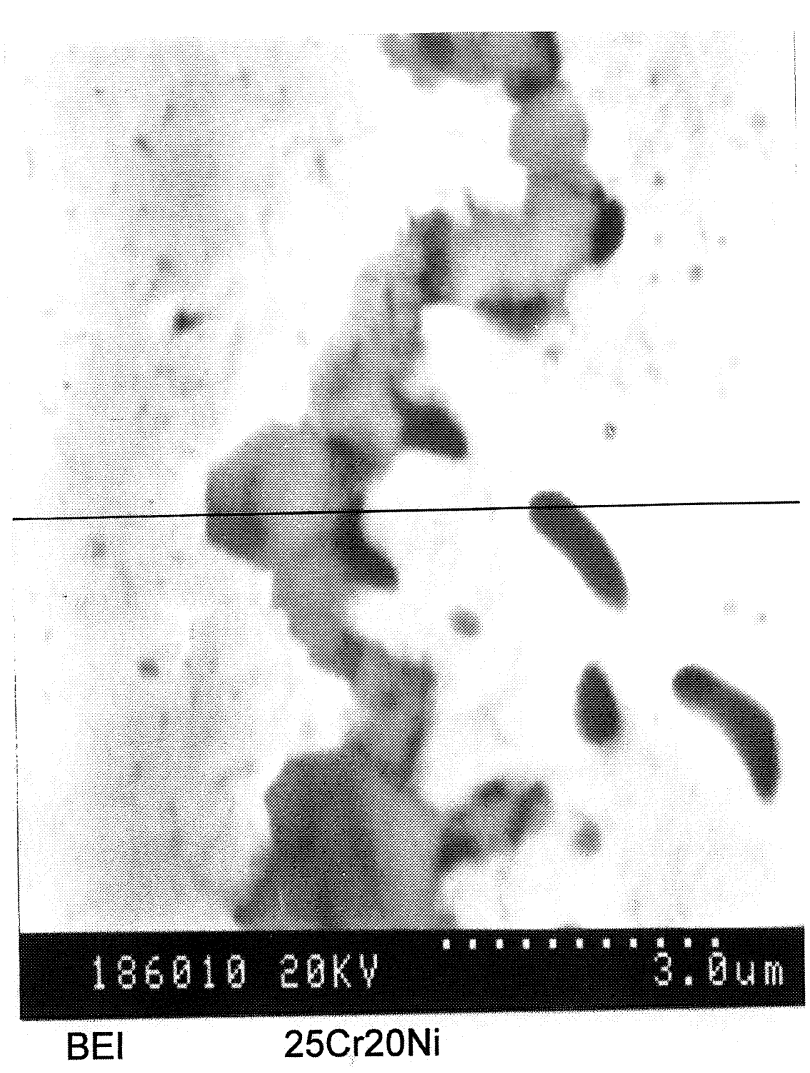

FIG. 5.- Detalle de la figura 3.

FIG. 5.-- Same than figure 3 at higher magnification.
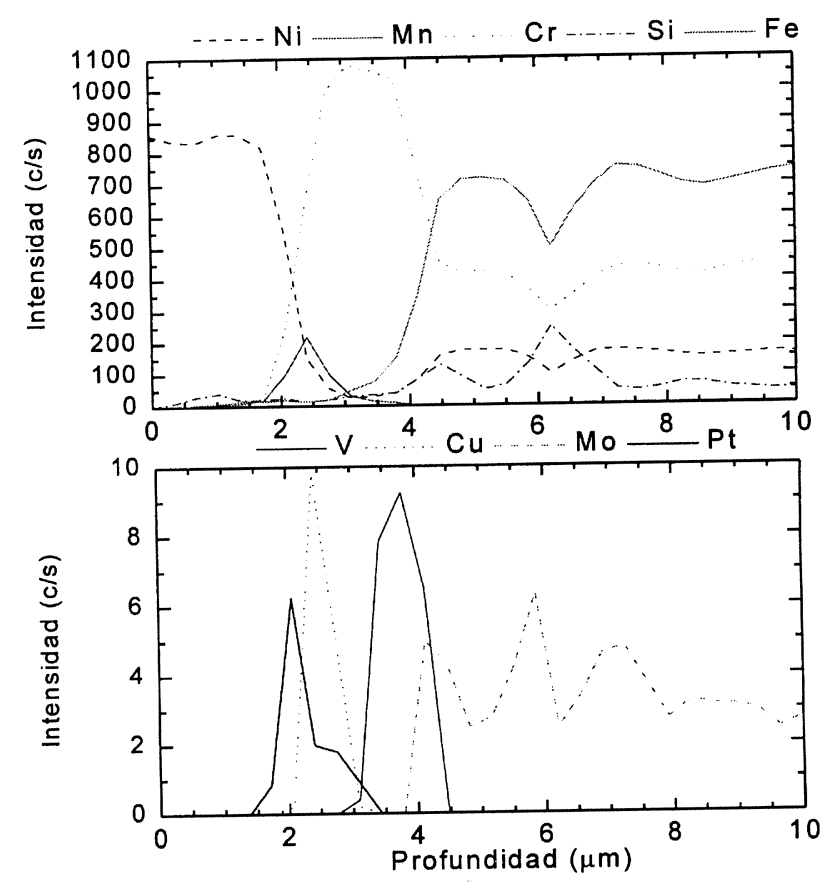

FIG. 6.- Análisis en línea de los elementos presentes en la figura 5 .

FIG. 6. - Line profile of elements found in figure 5.

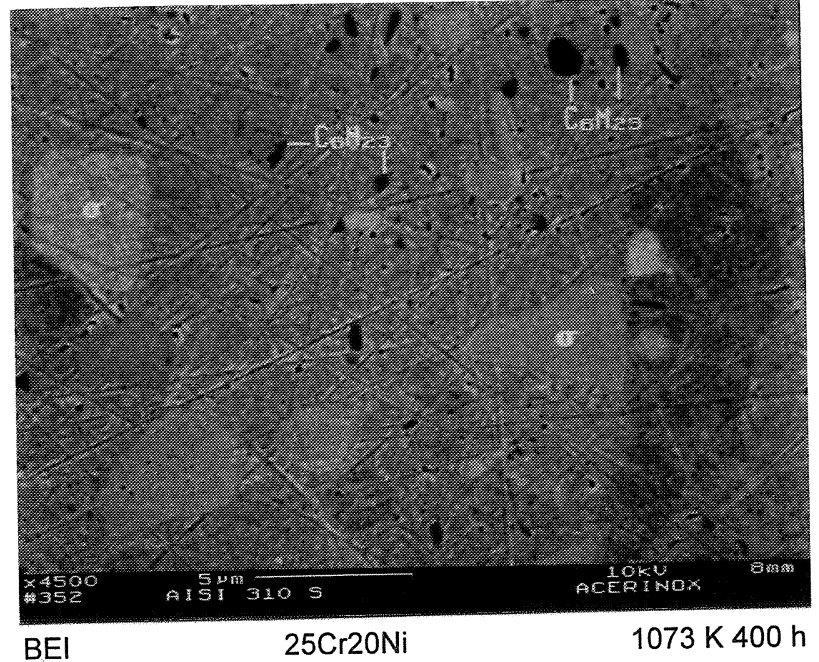

FIG. 7.- Sección transversal del acero tras tratamiento de envejecimiento. Se aprecian fases $\gamma$ y $\sigma$ y carburos $\mathrm{M}_{23} \mathrm{C}_{6}$

FIG. 7.- Cross section of the steel after ageing treatment. $\gamma$ and $\sigma$ phases and $M_{23} C_{6}$ carbides can be appreciated.

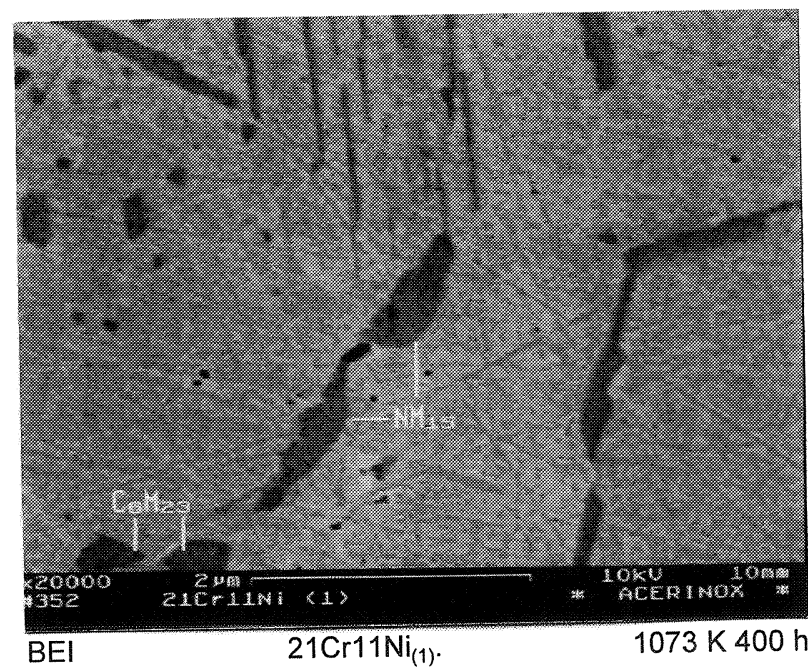

FIG. 8.- Sección transversal tras el tratamiento de envejecimiento. Se aprecian fase $\gamma$, carburos $\mathrm{M}_{23} \mathrm{C}_{6}$ y nitruros $\mathrm{M}_{15} \mathrm{~N}$.

FIG. 8. - Cross section of the steel after ageing treatment. $\gamma$-phase, $M_{23} C_{6}$ carbides and $M_{15} \mathrm{~N}$ nitrides can be seen.

en los tres aceros. El AISI $310 \mathrm{~S}$ sólo presenta carburos del tipo $\mathrm{M}_{23} \mathrm{C}_{6}$ muy ricos en cromo. Los aceros $21 \mathrm{Cr} 11 \mathrm{Ni}_{(1)}$ y $21 \mathrm{Cr} 11 \mathrm{Ni}_{(2)}$ presentan también estos carburos, pero en el material $21 \mathrm{Cr} 11 \mathrm{Ni}_{(1)}$ son muy relevantes los nitruros del tipo $\mathrm{M}_{15} \mathrm{~N}$ (ricos en cromo pero también en níquel y silico) y del tipo $\mathrm{M}_{8} \mathrm{~N}$ cerca de la superficie del material, mientras que en el $21 \mathrm{Cr} 11 \mathrm{Ni}_{(2)}$, los precipitados relevantes 


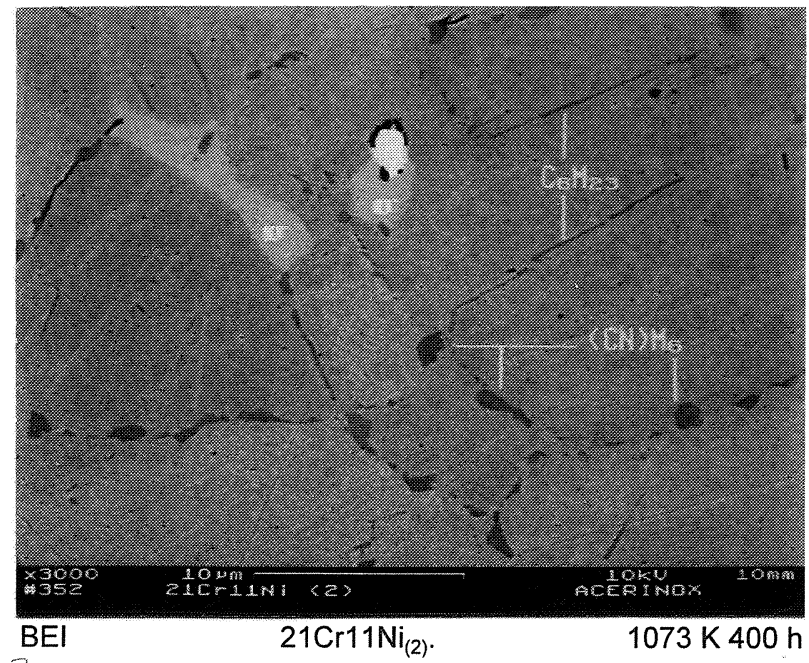

FIG. 9.- Sección transversal después del tratamiento de envejecimiento. Se aprecian fases $\gamma$ y $\sigma$, carburos $\mathrm{M}_{23} \mathrm{C}_{6}$ y carbonitruros $\mathrm{M}_{6}(\mathrm{CN})$. La partícula blanca corresponde a una inclusión de $\mathrm{CeO}_{2}$.

FIG. 9.- Cross section after ageing treatment. $\gamma$ and $\sigma$ phases, $M_{23} C_{6}$ carbides and $M_{6}(C N)$ carbonitrides can be appreciated. The white particle is a $\mathrm{CeO}_{2}$ inclusion. son los carbonitruros $\mathrm{M}_{6}(\mathrm{CN})$ ricos en cromo $\mathrm{y}$, además, en níquel y silicio.

Dado que las composiciones químicas de los aceros $21 \mathrm{Cr} 11 \mathrm{Ni}_{(1)}$ y $21 \mathrm{Cr} 11 \mathrm{Ni}_{(2)}$ son muy parecidas, que sólo se diferencian en los lantánidos (cabe pensar que estos no influyen en los mecanismos de formación de la fase $\sigma$ ) y en los niveles de nitrógeno y de silicio, es posible que en la no formación de $\sigma$ en el $21 \mathrm{Cr} 11 \mathrm{Ni}_{(1)}$ estén influyendo los relativamente menores niveles de silicio, pero sobre todo los más elevados niveles de nitrógeno, que a la vez que inhiben la formación de $\sigma$, potencian la formación de nitruros en el $21 \mathrm{Cr}_{11} 1 \mathrm{Ni}_{(1)}$, mientras que en el $21 \mathrm{Cr} 11 \mathrm{Ni}_{(2)}$, con niveles más bajos en nitrógeno, se forman, preferentemente, carbonitruros.

Ya ha sido comentado que, en general, el mejor comportamiento refractario lo tiene el AISI $310 \mathrm{~S}$, tanto a 1.373 como a $1.473 \mathrm{~K}$. No obstante, habría que tener en cuenta que el $21 \mathrm{Cr} 11 \mathrm{Ni}_{(1)}$ presenta prácticamente el mismo resultado que el AISI 310 $\mathrm{S}$ a $1.373 \mathrm{~K}$ y $\sin$ embargo no precipita fase $\sigma$ después de $400 \mathrm{~h}$ a $1.073 \mathrm{~K}$, lo que pudiera llegar a ser un factor determinante de la calidad en ciertas aplicaciones de ingeniería.

Con respecto a los precipitados encontrados, también tras el periodo de envejecimiento de $400 \mathrm{~h}$

TABLA VI.- Composiciones químicas comparadas (\% en masa): Matrices metálicas originales y fases $\sigma$ formadas $(400 \mathrm{~h} / 1.073 \mathrm{~K})$

TABLE VI.- Compared chemical compositions (mass \%): Original metallic matrix and developed $\sigma$ phases $(400 \mathrm{~h} / \mathrm{l}, 073 \mathrm{~K})$

\begin{tabular}{|c|c|c|c|c|c|}
\hline & \multicolumn{3}{|c|}{$25 \mathrm{Cr} 20 \mathrm{Ni}_{\text {(AISI } 310 \mathrm{~S})}$} & \multicolumn{2}{|l|}{$21 \mathrm{Cr} 11 \mathrm{Ni}_{(2)}$} \\
\hline & \multirow{2}{*}{$\begin{array}{l}\text { Matriz } \\
\text { original }\end{array}$} & \multicolumn{2}{|c|}{ Fase $\sigma$} & \multirow{2}{*}{$\begin{array}{l}\text { Matriz } \\
\text { original }\end{array}$} & \multirow{2}{*}{$\begin{array}{c}\text { Fase } \mathrm{o} \\
\overline{\mathrm{X}}\end{array}$} \\
\hline & & $\bar{X}$ & $\sigma_{n-1}$ & & \\
\hline $\mathrm{Si}$ & 1,02 & 1,79 & 0,05 & 1,88 & 3,00 \\
\hline $\mathrm{Cr}$ & 24,59 & 43,28 & 0,32 & 20,97 & 37,87 \\
\hline $\mathrm{Fe}$ & 53,97 & 45,55 & 0,41 & 64,93 & 53,97 \\
\hline $\mathrm{Ni}$ & 19,30 & 8,63 & 0,39 & 10,96 & 5,16 \\
\hline Mo & 0,19 & 0,75 & 0,08 & 0,10 & n.d. \\
\hline $\mathrm{Mn}$ & 0,39 & n.d & & 0,42 & n.d. \\
\hline $\mathrm{Cu}$ & 0,16 & n.d. & & 0,11 & n.d. \\
\hline Co & 0,15 & n.d. & & 0,23 & n.d. \\
\hline $\mathrm{V}$ & 0,09 & n.d. & & 0,12 & n.d. \\
\hline $\mathrm{N}$ & 0,025 & n.d. & & 0,137 & n.d. \\
\hline
\end{tabular}

Notas: i.- $\sigma_{25 \mathrm{Cr} 20 \mathrm{Ni}}\left[\mathrm{Cr}_{0,87} \mathrm{Fe}_{0,91} \mathrm{Ni}_{0,17} \mathrm{Si}_{0,04} \mathrm{Mo}_{0,01}\right]$.

ii.- $\sigma_{21 \mathrm{Cr} 11 \mathrm{Ni}(2)}\left[\mathrm{Cr}_{0,76} \mathrm{Fe}_{1,08} \mathrm{Ni}_{0,10} \mathrm{Si}_{0,06}\right]$.

iii.- n.d.: no determinado $(<0,1 \%)$.

iv.- Los resultados de $\sigma$ del $25 \mathrm{Cr} 20 \mathrm{Ni}$ son el promedio de seis análisis; en el $21 \mathrm{Cr} 11 \mathrm{Ni}_{(2)}$ sólo se han promediado dos. 
TABLA VII.- Cocientes entre elementos ( $\%$ en masa): Fase $\sigma / m a t r i z$ original

TABLE VII.- Element mass \% rates: $\sigma$ phase/original matrix

\begin{tabular}{|c|c|c|c|c|}
\hline & \multirow{2}{*}{$\begin{array}{c}25 \mathrm{Cr} 20 \mathrm{Ni} \\
\text { (AISI 310 S) }\end{array}$} & $21 \mathrm{Cr} 11 \mathrm{Ni}_{(2)}$ & $\overline{\mathrm{X}}$ & $\sigma_{\mathrm{n}-1}$ \\
\cline { 3 - 5 } & 1,75 & 1,60 & 1,66 & 0,15 \\
$\mathrm{Si}$ & 1,76 & 1,81 & 1,74 & 0,09 \\
$\mathrm{Cr}$ & 0,84 & 0,83 & 0,82 & 0,02 \\
$\mathrm{Fe}$ & 0,45 & 0,47 & 0,41 & 0,03 \\
$\mathrm{Ni}$ & 3,95 & n.d. & - & - \\
$\mathrm{Mo}$ & & & - & - \\
\hline
\end{tabular}

Notas: i.- * trabajando con $21 \mathrm{Cr} 11 \mathrm{Ni}$ con diferentes niveles de Ln's, C, N, a $1.023 \mathrm{~K}$ entre 2.600 y $9.700 \mathrm{~h}$ de mantenimiento.

ii.- n.d.: no determinado.

TABLA VIII.- Composiciones químicas (\% at.) y estequiometrías calculadas de carburos, carbonitruros y nitruros precipitados $(400 \mathrm{~h} / 1.073 \mathrm{~K})$

TABLE VIII.- Chemical composition (at. \%) and calculated stoichiometry of precipitated carbides, carbonitrides and nitrides $(400 \mathrm{~h} / 1,073 \mathrm{~K})$

\begin{tabular}{|c|c|c|c|c|c|c|c|c|c|c|c|c|c|}
\hline Materiales & Situación & \multicolumn{2}{|c|}{ Estadística } & $\mathrm{C}$ & $\mathrm{N}$ & $\mathrm{Si}$ & $\mathrm{V}$ & $\mathrm{Cr}$ & $\mathrm{Fe}$ & $\mathrm{Ni}$ & Mo & $\mathrm{Cu}$ & Estequiometría \\
\hline \multirow[t]{2}{*}{$\begin{array}{l}25 \mathrm{Cr} 20 \mathrm{Ni} \\
\text { (AISI } 310 \mathrm{~S} \text { ) }\end{array}$} & & $n=3$ & $\overline{\mathrm{X}}$ & 20,13 & - & 0,42 & 0,24 & 59,90 & 16,02 & 2,66 & 0,50 & - & \multirow[t]{2}{*}{$\begin{array}{l}\mathrm{M}_{78,87} \mathrm{C}_{20,13} \\
\left(\mathrm{M}_{23,81} \mathrm{C}_{6}\right)\end{array}$} \\
\hline & & & $\sigma_{\mathrm{n}-1}$ & 0,86 & - & 0,48 & 0,04 & 0,69 & 0,70 & 0,31 & 0,06 & - & \\
\hline \multirow{8}{*}{$21 \mathrm{Cr} 11 \mathrm{Ni}_{(1)}$} & Centro & $n=2$ & $\overline{\mathrm{X}}$ & 20,91 & - & 0,99 & 0,20 & 60,65 & 13,58 & 2,61 & 0,68 & 0,24 & $\begin{array}{l}\mathrm{M}_{79,09} \mathrm{C}_{20,91} \\
\left(\mathrm{M}_{22,69} \mathrm{C}_{6}\right)\end{array}$ \\
\hline & Centro & $\mathrm{n}=2$ & $\overline{\mathrm{X}}$ & - & 6,22 & 13,67 & 0,94 & 43,81 & 7,41 & 25,36 & 0,94 & 1,65 & $\begin{array}{c}\mathrm{M}_{93,79} \mathrm{~N}_{6,21} \\
\left(\mathrm{M}_{15,10} \mathrm{~N}\right)\end{array}$ \\
\hline & \multirow[t]{2}{*}{ Superficie } & \multirow[t]{2}{*}{$n=6$} & $\overline{\mathrm{x}}$ & - & 11,10 & 8,51 & 0,64 & 51,95 & 17,34 & 10,11 & 0,35 & - & \multirow[t]{2}{*}{$\begin{array}{c}\mathrm{M}_{88,90} \mathrm{~N}_{11,10} \\
\left(\mathrm{M}_{8,00} \mathrm{~N}\right)\end{array}$} \\
\hline & & & $\sigma_{n-1}$ & - & 0,81 & 0,11 & 0,19 & 0,65 & 0,47 & 0,67 & 0,03 & - & \\
\hline & \multirow[t]{2}{*}{ Superficie } & \multirow[t]{2}{*}{$n=3$} & $\overline{\mathrm{X}}$ & 20,43 & - & 0,90 & - & 57,07 & 17,98 & 2,78 & 0,56 & - & \multirow[t]{2}{*}{$\begin{array}{l}\mathrm{M}_{79,57} \mathrm{C}_{20,43} \\
\left(\mathrm{M}_{23,37} \mathrm{C}_{6}\right)\end{array}$} \\
\hline & & & $\sigma_{n-1}$ & 2,54 & - & 0,52 & - & 4,14 & 5,11 & 0,39 & 0,10 & - & \\
\hline & \multirow[t]{2}{*}{ Superficie } & \multirow[t]{2}{*}{$\mathrm{n}=3$} & $\overline{\mathrm{X}}$ & - & 5,98 & 12,49 & 0,83 & 42,37 & 11,54 & 25,89 & 0,70 & - & \multirow[t]{2}{*}{$\begin{array}{l}\mathrm{M}_{94,02} \mathrm{~N}_{5,98} \\
\left(\mathrm{M}_{15,72} \mathrm{~N}\right)\end{array}$} \\
\hline & & & $\sigma_{\mathrm{n}-1}$ & - & 0,84 & 1,95 & 0,07 & 3,11 & 5,04 & 0,58 & 0,14 & - & \\
\hline \multirow{3}{*}{$\begin{array}{l}21 \mathrm{Cr} 11 \mathrm{Ni}_{(2)} \\
0,01 \mathrm{LN} \text { 's }\end{array}$} & \multirow[t]{2}{*}{ Abundantes } & \multirow[t]{2}{*}{$n=6$} & $\overline{\mathrm{X}}$ & 6,56 & 7,54 & 11,42 & 2,76 & 41,00 & 7,56 & 22,66 & 0,44 & - & \multirow[t]{2}{*}{$\begin{array}{c}\mathrm{M}_{85,84}(\mathrm{CN})_{14.6} \\
\mathrm{M}_{6,06}(\mathrm{CN})\end{array}$} \\
\hline & & & $\sigma_{n-1}$ & 1,66 & 1,29 & 1,74 & 0,70 & 3,35 & 1,13 & 1,88 & 0,09 & - & \\
\hline & Escasas & $\mathrm{n}=2$ & $\overline{\mathrm{x}}$ & 20,44 & - & 1,56 & 0,36 & 58,23 & 16,44 & 2,60 & 0,30 & - & $\begin{array}{l}\mathrm{M}_{79,56} \mathrm{C}_{20,44} \\
\left(\mathrm{M}_{23,35} \mathrm{C}_{6}\right)\end{array}$ \\
\hline
\end{tabular}

a $1.073 \mathrm{~K}$, podemos hacer las siguientes consideraciones:

La tabla IX presenta las estequiometrías de los carburos $\mathrm{M}_{23} \mathrm{C}_{6}$, deducidas de los datos de análisis EDX, normalizados a 23 átomos metálicos, compa- rándolas con las de los carburos, también $\mathrm{M}_{23} \mathrm{C}_{6}$, obtenidas por otros autores, (2-6), en diferentes aleaciones de aceros inoxidables.

Hay que señalar la diferente composición de los carburos $\mathrm{M}_{23} \mathrm{C}_{6}$ encontrados en el acero 
TABLA IX.- Estequiometrías calculadas para $\mathrm{M}_{23} \mathrm{C}_{6}$

TABLE IX.- Calculated stoichiometries of $M_{23} C_{6}$

\begin{tabular}{|c|c|c|c|}
\hline & Aleación & Estequiometrías $\mathrm{M}_{23} \mathrm{C}_{6}$ & \\
\hline \multirow{4}{*}{$\begin{array}{l}\text { Trabajo } \\
\text { presente }\end{array}$} & $\left.25 \mathrm{Cr} 20 \mathrm{Ni}_{(\mathrm{AISI}} 310 \mathrm{~S}\right)$ & $\mathrm{Cr}_{17,28} \mathrm{Fe}_{4,62} \mathrm{Mo}_{0,14} \mathrm{Ni}_{0,77} \mathrm{Si}_{0,12} \mathrm{~V}_{0,07}$ & $\mathrm{C}_{6}$ \\
\hline & & $\begin{array}{l}\mathrm{Cr}_{17,67} \mathrm{Fe}_{3,96} \mathrm{Mo}_{0,20} \mathrm{Ni}_{0,76} \mathrm{Si}_{0,29} \mathrm{~V}_{0,06} \mathrm{Cu}_{0,07} \\
\text { (Centro del espesor) }\end{array}$ & $\mathrm{C}_{6}$ \\
\hline & $21 \mathrm{Cr} 11 \mathrm{Ni}_{(1)}$ & $\begin{array}{l}\mathrm{Cr}_{16,55} \mathrm{Fe}_{5,22} \mathrm{Mo}_{0,16} \mathrm{Ni}_{0,81} \mathrm{Si}_{0,26} \\
\text { (Bordes) }\end{array}$ & $\mathrm{C}_{6}$ \\
\hline & $21 \mathrm{Cr} 11 \mathrm{Ni}_{(2)}$ & $\mathrm{Cr}_{16,85} \mathrm{Fe}_{4,76} \mathrm{Mo}_{0,09} \mathrm{Ni}_{0,75} \mathrm{Si}_{0,45} \mathrm{~V}_{0,17}$ & $\mathrm{C}_{6}$ \\
\hline \multirow{5}{*}{$\begin{array}{l}\text { Otros } \\
\text { autores }\end{array}$} & (2) $21 \mathrm{Cr} 11 \mathrm{Ni}$ & $\mathrm{Cr}_{20,0} \mathrm{Fe}_{2,4} \mathrm{Ni}_{0,6}$ & $\mathrm{C}_{6}$ \\
\hline & (4) $\left.17 \mathrm{Cr} 12 \mathrm{Ni} 2 \mathrm{Mo}_{(\mathrm{AISI}} 316\right)$ & $\mathrm{Cr}_{17,0} \mathrm{Fe}_{4,5} \mathrm{Mo}_{1,5}$ & $\mathrm{C}_{6}$ \\
\hline & (5) $17 \mathrm{Cr} 12 \mathrm{Ni} 2 \mathrm{Mo}_{(\text {AISI } 316 \mathrm{~L})}$ & $\mathrm{Cr}_{16,0} \mathrm{Fe}_{5,0} \mathrm{Mo}_{2,0}$ & $\mathrm{C}_{6}$ \\
\hline & (6) $\left.16 \mathrm{Cr} 10 \mathrm{Ni} 2 \mathrm{Mo}_{(\mathrm{AISI}} 316 \mathrm{~L} 316 \mathrm{LN}\right)$ & $\mathrm{Cr}_{16,2} \mathrm{Fe}_{4,4} \mathrm{Mo}_{1,8} \mathrm{Ni}_{0,6}$ & $\mathrm{C}_{6}$ \\
\hline & (7) $21 \mathrm{Cr} 10 \mathrm{Ni}_{(\mathrm{AISI} 308)}$ & $\mathrm{Cr}_{18,2} \mathrm{Fe}_{4,2} \mathrm{Ni}_{0,6}$ & $\mathrm{C}_{6}$ \\
\hline
\end{tabular}

$21 \mathrm{Cr} 11 \mathrm{Ni}_{(1)}$, dependiendo de que estén situados en el interior o próximos a los bordes. Los carburos próximos al borde, es decir cerca de las superficies del acero, son relativamente más pobres en cromo y más ricos en hierro, níquel y molibdeno, probablemente debido a los gradientes de composición química originados por la formación superficial de óxidos ricos en $\mathrm{Cr}_{2} \mathrm{O}_{3}$, como ya se ha comentado para el caso de la ausencia de fase $\sigma$ en las cercanías de los bordes en el material 25Cr20Ni (AISI $310 \mathrm{~S}$ ).

Todos los carburos $\mathrm{M}_{23} \mathrm{C}_{6}$ de las tres aleaciones ensayadas tienen ciertos niveles de níquel (alrededor de 0,8 átomos sobre 23) y niveles residuales de molibdeno y silicio (alrededor de 0,1 y 0,2 átomos respectivamente sobre 23 ). La presencia de níquel (0,6 átomos sobre 23$)$ es detectada por algunos autores, $(2$ y 6$)$, en austenitas sin molibdeno, y por otros (5) en austenitas con molibdeno. En general, puede decirse que los autores que analizan austenitas con molibdeno (AISI 316, L, LN) encuentran una importante participación del molibdeno en los carburos $\mathrm{M}_{23} \mathrm{C}_{6}$ (entre 1,5 y 2,0 átomos de molibdeno de los 23 de la estequiometría). Novak (7) señala que el $\mathrm{M}_{23} \mathrm{C}_{6}$ es primariamente $\mathrm{Cr}_{23} \mathrm{C}_{6}$ en el que el hierro (o el molibdeno) puede sustituir parcialmente al cromo y que la extensión de la substitución parece depender de las aleaciones y de las condiciones de precipitación. En nuestras experiencias, ninguna de las aleaciones está aleada con molibdeno (sólo existen niveles residuales de 0,1 a $0,2 \%$ en masa) y sin embargo se ha apreciado que la participación del molibdeno en los carburos $\mathrm{M}_{23} \mathrm{C}_{6}$ es significativa y proporcional al contenido de este elemento en la aleación.
Dacasa et al. (8) indican que las condiciones de precipitación -tiempo y temperatura- influyen en la composición de los carburos. Según estos autores, es posible establecer un valor constante de 4,5 para el cociente $\mathrm{Cr} / \mathrm{Fe}$ (\% masa) de los carburos $\mathrm{M}_{23} \mathrm{C}_{6}$ formados en un $18 \mathrm{Cr} 10 \mathrm{Ni}$ mantenido $400 \mathrm{~h}$ a 1.073 K. Dicho cociente se establece para carburos en los que, según citan, sólo cromo y hierro constituyen la parte metálica, lo que no ocurre en nuestro caso, donde la composición de los carburos podría estar condicionada, no sólo por la composición inicial de la matriz metálica y de los factores temperatura y tiempo sino también, por todas las condiciones de un equilibrio en el que no sólo precipitan $\operatorname{los} \mathrm{M}_{23} \mathrm{C}_{6}$ sino que también se forman fases (como la $\sigma$ ) y otros precipitados como nitruros y carbonitruros. Sin embargo, Hall et al. (5), trabajando con aleaciones tipo AISI 316, señalan que la composición química de los precipitados tipo $\mathrm{M}_{23} \mathrm{C}_{6}$ no cambia ni con la temperatura ni con el tiempo; si bien es cierto que estos autores no tienen evidencias de ninguna formación de fases ni de precipitados que no fuesen los carburos $\mathrm{M}_{23} \mathrm{C}_{6}$.

La abundante precipitación de carbonitruros $\mathrm{M}_{6}(\mathrm{CN})$ en el $21 \mathrm{Cr} 11 \mathrm{Ni}_{(2)}$ y la escasa presencia de los $\mathrm{M}_{23} \mathrm{C}_{6}$ diferencia claramente el comportamiento de esta aleación con respecto a la $21 \mathrm{Cr} 11 \mathrm{Ni}_{(1)}$, en la que predominan los precipitados basados en el $\mathrm{M}_{23} \mathrm{C}_{6}$ y $\mathrm{M}_{15} \mathrm{~N}$. Las principales diferencias se resumen en la tabla $X$.

A la vista de los datos de la tabla $\mathrm{X}$ todo parece indicar que en el $21 \mathrm{Cr} 11 \mathrm{Ni}_{(1)}$, los elevados niveles de nitrógeno $-1.780 \mathrm{ppm}$ - a la vez que inhiben la 
TABLA X.- Diferencias de precipitados, de fases y de composiciones químicas de los materiales $21 \mathrm{Cr} 11 \mathrm{Ni}$ $(400 \mathrm{~h} / 1.073 \mathrm{~K})$

TABLE X.- Relevant differences of precipitates, phases and chemical composition of $21 \mathrm{Cr} 11 \mathrm{Ni}$ materials $(400 \mathrm{~h} / 1,073 \mathrm{~K})$

\begin{tabular}{|l|c|c|c|c|c|}
\hline & \multicolumn{3}{|c|}{ \% en masa } & Precipitados $400 \mathrm{~h} / 1.073 \mathrm{~K}$ & $\sigma$ \\
\cline { 1 - 4 } Aleación & $\mathrm{N}$ & $\mathrm{Si}$ & $\mathrm{C}$ & & \\
\hline $21 \mathrm{Cr} 11 \mathrm{Ni}_{(1)}$ & 0,1780 & 1,47 & 0,087 & $\left(\mathrm{M}_{15} \mathrm{~N}\right),\left(\mathrm{M}_{23} \mathrm{C}_{6}\right)$ & No se forma \\
$21 \mathrm{Cr} 11 \mathrm{Ni}_{(2)}$ & 0,1367 & 1,88 & 0,075 & $\mathrm{M}_{6}(\mathrm{CN}),\left(\mathrm{M}_{23} \mathrm{C}_{6}\right)$ & Sí se forma \\
\hline
\end{tabular}

formación de fase $\sigma$, estimulan la formación de nitruros. Los niveles de nitrógeno en el $21 \mathrm{Cr} 11 \mathrm{Ni}_{(2)}$ $-1.367 \mathrm{ppm}$ - parece que no son suficientes para evitar la formación de fase $\sigma$ y, a la vez, parece que no son suficientes para la formación de nitruros en las condiciones experimentadas, siendo el carbonitruro $\mathrm{M}_{6}(\mathrm{CN})$ el compuesto favorecido.

Los carburos $\mathrm{M}_{6} \mathrm{C}$ se han asociado (9) a inoxidables al molibdeno y se ha establecido una hipótesis de génesis a partir de los carburos $\mathrm{M}_{23} \mathrm{C}_{6}$ :

$$
\mathrm{M}_{23} \mathrm{C}_{6} \rightarrow(\mathrm{FeCr})_{21} \mathrm{Mo}_{2} \mathrm{C}_{6} \stackrel{+\mathrm{Mo}}{\rightarrow} \mathrm{M}_{6} \mathrm{C}
$$

Una típica composición de estos $\mathrm{M}_{6} \mathrm{C}$ podría ser $(\mathrm{Cr}, \mathrm{Fe})_{3,5} \mathrm{Mo}_{2.5} \mathrm{C}$, citada en (9).

Otros autores (10) presentan evidencias de que el nitrógeno se disuelve en los carburos $\mathrm{M}_{6} \mathrm{C}$ precipitados en austenitas ricas en molibdeno $(5 \%$ en masa) y nitrógeno variable (entre 0,069 y $0,247 \%$ ). En el presente trabajo no se ha obtenido ninguna evidencia de evoluciones desde un $\mathrm{M}_{23} \mathrm{C}_{6}$ a un $\mathrm{M}_{6} \mathrm{C}$, ni de este a un $\mathrm{M}_{6}(\mathrm{CN})$. La estequiometría $\mathrm{Cr}_{2,87} \mathrm{Fe}_{0,53} \mathrm{Ni}_{1,58} \mathrm{Si}_{0,80} \mathrm{~V}_{0,19} \mathrm{Mo}_{0,03}(\mathrm{CN})$ podría agruparse como $(\mathrm{Cr}, \mathrm{Fe})_{3,4}(\mathrm{Ni}, \mathrm{Si})_{2,4}(\mathrm{~V}, \mathrm{Mo})_{0,3}(\mathrm{CN})$, de modo que el agrupamiento $(\mathrm{Cr}, \mathrm{Fe})$ sería significativamente el mismo que en (9), y donde el molibdeno constituyente del $(\mathrm{Cr}, \mathrm{Fe})_{3,5} \mathrm{Mo}_{2,5} \mathrm{C}$ estaría reemplazado fundamentalmente por el níquel (y el silicio) con una muy significativa presencia del vanadio, habida cuenta de que en la aleación del $21 \mathrm{Cr} 11 \mathrm{Ni}_{(2)}$ los niveles de vanadio son residuales $(0,12 \%$ en masa).

\section{CONCLUSIONES}

Del estudio comparativo de la oxidación de los tres inoxidables austeníticos $\left(25 \mathrm{Cr} 20 \mathrm{Ni}, 21 \mathrm{Cr} 11 \mathrm{Ni}_{(1)}\right.$ y $\left.21 \mathrm{Cr} 11 \mathrm{Ni}_{(2)}\right)$ se concluye que el $25 \mathrm{Cr} 20 \mathrm{Ni}$-AISI $310 \mathrm{~S}-$ está mejor adaptado al uso refractario, tanto a 1.373 como a $1.473 \mathrm{~K}$. A $1.373 \mathrm{~K}$ tiene un com- portamiento prácticamente idéntico el $21 \mathrm{Cr} 11 \mathrm{Ni}_{(1)}$ microaleado con $0,036 \%$ de lantánidos, y algo peor el $21 \mathrm{Cr} 11 \mathrm{Ni}_{(2)}$ con un $0,010 \%$ de lantánidos.

Según se deduce del estudio de los materiales en la oxidación al aire a $1.323 \mathrm{~K}(2 \mathrm{~h})$, los tres aceros basan su resistencia a este tipo de oxidación en la formación dẹ capas de $\mathrm{Cr}_{2} \mathrm{O}_{3}$ por debajo de las cuales se presentan numerosos precipitados ricos en $\mathrm{SiO}_{2}$, a modo de cuasi barrera en la interfase óxidometal, así como precipitados internos también de $\mathrm{SiO}_{2}$, más abundantes en los materiales $21 \mathrm{Cr} 11 \mathrm{Ni}$. Las barreras protectoras se completan con capas cristalinas de $\mathrm{MnCr}_{2} \mathrm{O}_{4}$ creciendo por encima de las de $\mathrm{Cr}_{2} \mathrm{O}_{3}$, en la interfase óxido-atmósfera.

El mejor comportamiento refractario a $1.323 \mathrm{~K}$ $(2 \mathrm{~h}), 1.373$ y $1.473 \mathrm{~K}(50 \mathrm{~h})$ del AISI $310 \mathrm{~S}$ contrasta con una relativamente alta precipitación de fase $\sigma$ (alrededor de un $10 \%$ vol.) en un tratamiento térmico de envejecimiento de $400 \mathrm{~h}$ a $1.073 \mathrm{~K}$. En el material $21 \mathrm{Cr} 11 \mathrm{Ni}_{(1)}$ no se forma fase $\sigma$ y es muy escasa la presencia de la misma en el $21 \mathrm{Cr} 11 \mathrm{Ni}_{(2)}$.

En la inhibición de formación de fase $\sigma$, en los materiales $21 \mathrm{Cr} 11 \mathrm{Ni}$, están sin duda los menores niveles de aleación con respecto al $25 \mathrm{Cr} 20 \mathrm{Ni}$-especialmente en el elemento cromo-, pero fundamentalmente en la aleación de nitrógeno de los $21 \mathrm{Cr} 11 \mathrm{Ni}$.

Esta misma presencia de nitrógeno es la responsable de la formación de nitruros de estequiometría $\mathrm{M}_{15 \sim 16} \mathrm{~N}$ en $21 \mathrm{Cr} 11 \mathrm{Ni}_{(1)}$ y de carbonitruros $\mathrm{M}_{6}(\mathrm{CN})$ en $21 \mathrm{Cr} 11 \mathrm{Ni}_{(2)}$. En los tres materiales se forman, durante el envejecimiento, carburos tipo $\mathrm{M}_{23} \mathrm{C}_{6}$, siendo junto a la fase $\sigma$ los únicos precipitados en el $25 \mathrm{Cr} 20 \mathrm{Ni}$. Sin embargo, son relativamente escasos en $21 \mathrm{Cr}_{11 \mathrm{Ni}_{(1)}}$-donde abundan los nitruros- $\mathrm{y}$ muy escasos en $21 \mathrm{Cr} 11 \mathrm{Ni}_{(2)}$ en la que predominan los carbonitruros $\mathrm{M}_{6}(\mathrm{CN})$. Las diferencias de precipitados de los $21 \mathrm{Cr} 11 \mathrm{Ni}$ deben ser imputables a los diferentes niveles de nitrógeno: para el $0,18 \% \mathrm{~N}$ del $21 \mathrm{Cr} 11 \mathrm{Ni}_{(1)}$ dominan los nitruros, y para el 0,14 $\% \mathrm{~N}$ del $21 \mathrm{Cr} 11 \mathrm{Ni}_{(2)}$ se forman carbonitruros. 


\section{REFERENCIAS}

(1) Botella, J. Tesis Doctoral. Universidad Complutense de Madrid, 1996.

(2) Yu, M., Sandström, R., Lehtinen, B. y Westman, C. Scand. J. Metall. 16, 1987: 154-163.

(3) Kautz, H.R. y Gerlach, H. Arch. Eisenhuettenwes., 39 (2) $1968: 151$.

(4) Weiss, B. y StickleR, R. Metall. Trans., 3, 1972: 851.

(5) Hall, E.L., Bryant, C.L. Metall. Trans. A. 15A, 1984: 793-811.
(6) VITEK, J.M. y DAVID, S.A. Metall. Trans. A., 18A, 1987: 1185-1201.

(7) Novak, C.J. Handbook of Stainless steels. Ed. Pecker and Bernstein. McGraw Hill Book Co. Nueva York, Cap. 4, 1977: 4.41.

(8) Dacasa, C., Nileshwar, V.B. y Melford, D.A. J. Iron Steel Inst. London, 207.pt.10, 1969: 1325.

(9) Goldsmidt, H. Intersticial Alloys. Plenum Ores. Nueva York, 1967.

(10) Thier, H., Baumel, A. y Schmidtmann, E. Arch. Eisenhuetenwes., 40 (4), 1969: 333. 